\title{
SD, Rat Strain
}

National Cancer Institute

\section{Source}

National Cancer Institute. SD, Rat Strain. NCI Thesaurus. Code C76189.

Derived from Wistar rats at Sprague-Dawley farms, this rat strain is characterized by a calm temperament which lends itself to ease of handling. This rat strain has the following anatomical features: absent gallbladder, a one-lobed left lung and a four-lobed right lung, the inability to vomit, and the production of dark colored eye secretions during periods of stress. The SD rat has good breeding performance and a lifespan of 2.5-3.5 years. 